\title{
ESTIMATION OF AMOUNT OF SCATTERED NEUTRONS AT DEVICES PFZ AND GIT-12 BY MCNP SIMULATIONS
}

\author{
Ondřej Šíla*, Pavel Kubeš, Josef Kravárik, Karel Řezáč, Daniel Klír, \\ JAKUB CIKHARDT
}

\author{
Department of physics, Faculty of Electrical Engineerging, Czech Technical University in Prague, Czech Republic \\ * corresponding author: silaondr@fel.cvut.cz
}

\begin{abstract}
Our work is dedicated to pinch effect occurring during current discharge in deuterium plasma, and our results are connected with two devices - plasma focus PFZ, situated in the Faculty of Electrical Engineering, CTU, Prague, and Z-pinch GIT-12, which is situated in the Institute of High Current Electronics, Tomsk. During fusion reactions that proceed in plasma during discharge, neutrons are produced. We use neutrons as instrument for plasma diagnostics. Despite of the advantage that neutrons do not interact with electric and magnetic fields inside device, they are inevitably scattered by materials that are placed between their source and probe, and information about plasma from which they come from is distorted. For estimation of rate of neutron scattering we use MCNP code.
\end{abstract}

KEYWORDS: plasma focus, pinch effect, D-D reaction, MCNP.

\section{INTRODUCTION}

One of the most remarkable phenomena of plasma physics is pinch effect which proceeds if sufficiently high current passes through plasma. Such high current generates magnetic field force that compresses current layer radially - that leads to great increase of temperature in place of pinched current. If plasma is made of suitable gas like deuterium or tritium, we can observe fusion reactions during pinch effect.

Plasma focus is device that is mainly composed of two cylindrical electrodes placed in vessel which is filled by isotope of hydrogen (mostly deuterium). Between these electrodes current layer is formed (in most cases by discharge of capacitors) and moves in front of inner electrode where is compressed, and pinch effect proceeds. In some cases, there is another cylindrical electrode placed in front of inner electrode, and with purpose to support pinch effect this experimental setup appears for instance at PFZ device [1].

Device GIT-12 is situated in the Institute of High Current Electronics, Tomsk. Operation of this device is based on principle of gas-puff.

Both mentioned devices have vessel filled with deuterium, so during discharge $\mathrm{D}-\mathrm{D}$ reactions proceed within. Detection of D-D fusion neutrons is performed using scintillation probes with scintillator Bicron BC-408 [5].

For estimating of neutron energy we use time-offlight method. Because of the fact that neutrons from $\mathrm{D}-\mathrm{D}$ fusion reaction have velocity approximately $0.72 c$, we use simple non-relativistic equation for estimating neutron energy $E_{\mathrm{n}}$ from its velocity $v_{\mathrm{n}}$,

$$
E_{\mathrm{n}}=\frac{1}{2} m v_{\mathrm{n}}^{2} .
$$

\section{MCNP RESULTS}

MCNP (abbreviation for "Monte Carlo N-Particle") is being used for calculating of energies and positions of variety of particles (for instance electrons, photons, neutrons and positrons) during and after their transport from place A to place B, if they interact with specific materials which have unique shape. The main problem of defying parameters for simulation in MCNP is to define sufficiently geometry of experimental setup to get as realistic model as possible. User of this program creates input file where considered geometry, materials, particle source, type of results and number of iterations are defined [3].

\subsection{Parameters of OUR Simulations}

We created MCNP input file for devices PFZ and GIT-12. In both cases, we tried to define geometry of problem sufficiently similar to reality. Two dimensional view of our considered models is pictured in Figs. 1 and 2

In our simulations, we used mono-energetic point source with mean energy of $\mathrm{D}-\mathrm{D}$ fusion neutron $2.45 \mathrm{MeV}$. We placed this source to place which we considered as the most probable for production of fusion neutrons (we are able to determine this place with inaccuracy circa $5 \mathrm{~cm} \mathrm{[2]).} \mathrm{ENDF} \mathrm{cross} \mathrm{section} \mathrm{data} \mathrm{li-}$ braries were used to simulate neutron transport in our simulation. The type of result of simulation is defined by so called tally card. We chose tally F4 card, so our result was average energy of neutrons in cell which we defined as cylindrical and of the same dimensions as our scintillator. We located such cell to the same position and distance as the scintillation probe in our experiment. It is quite important to choose sufficiently great number of iterations of simulation to get results 


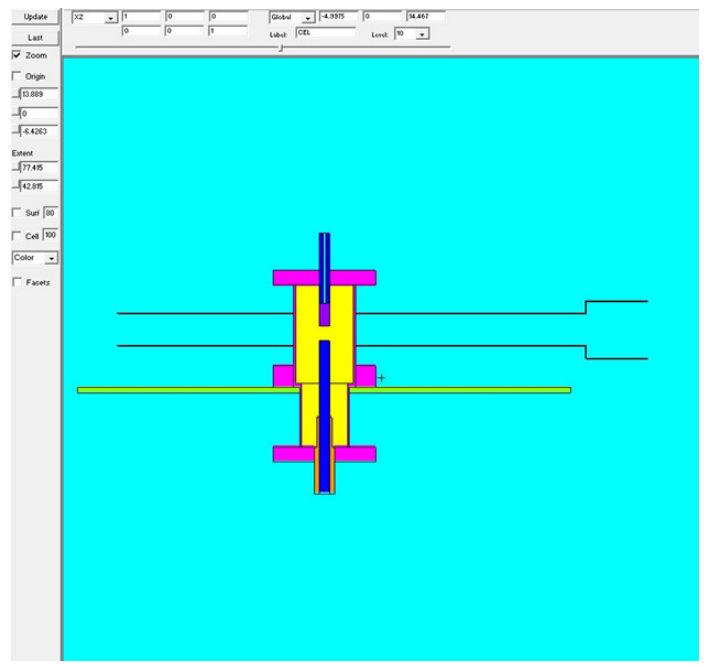

Figure 1. Parts of PFZ device which were implemented to MCNP input file.

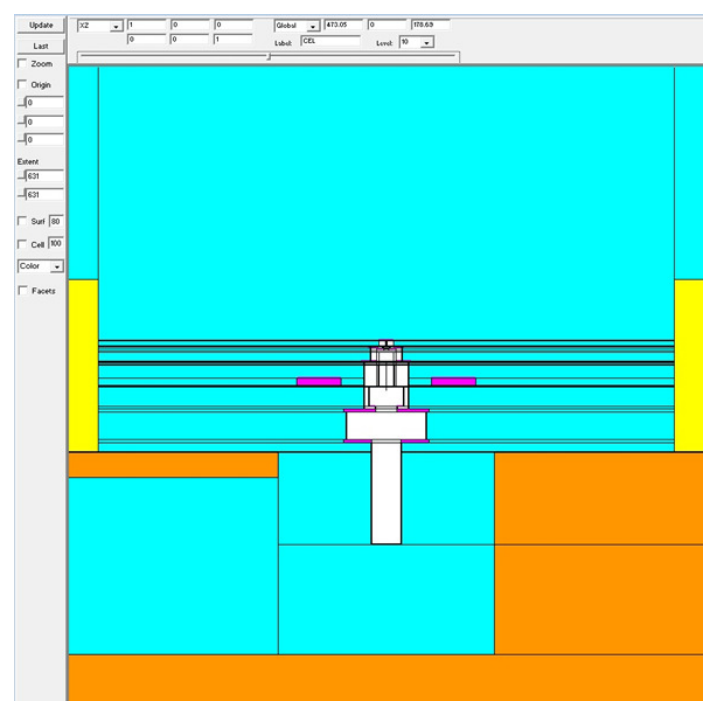

Figure 2. Parts of GIT-12 device which we considered in MCNP simulation.

accurate enough. For all our mentioned cases, $5 \times 10^{7}$ iterations gave us acceptably reasonable results.

When creating geometry of problem, there is a question whether floor and walls should be included to geometry or not. For each of these two devices we have different answer. In case of PFZ it is not necessary to take walls and floor into account because neutrons reflected from them do not manage to reach probe in the time range of observed neutron pulse. It is caused by the fact that in experimental setup of PFZ we have probes distanced not more than 5 meters from source of fusion neutrons. On the other hand, GIT-12 is device in which probes are placed in much greater distances from the place of production of fusion neutrons and it is very expectable that this reflected neutrons appear in observed neutron signal, so it is necessary to include floor and walls into geometry 4 .

We performed our simulations in version MCNP5 of this program.

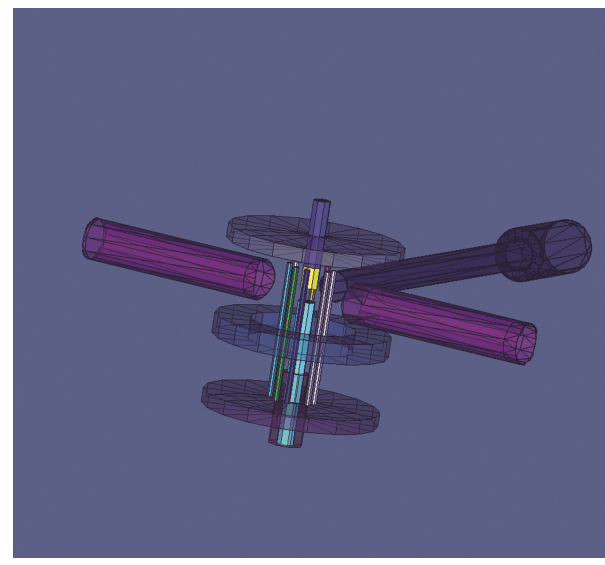

Figure 3. 3-D view of parts of PFZ device which were implemented to MCNP input file.

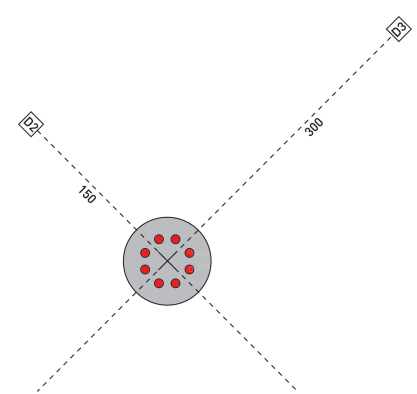

Figure 4. Deployment of radial detectors D2 and D3.

\subsection{MCNP RESUlTS FOR PFZ DEVICE}

\subsubsection{NEUTRON ENERGY SPECTRUM IN PLACE OF THREE SCINTILLATION PROBES IN PFZ EXPERIMENTAL SETUP}

We calculated neutron energy spectrum in three places where we put probes in our experimental setup. Deployment of detectors illustrates Fig. 4

In the Fig. 5, we present the results of our simulation.

We can see that the greatest number of scattered neutrons is observed in probe D1. We attribute this result to the fact that neutrons flying in axial direction have to overcome substantially thicker layer of metal than neutrons flying in radial direction, so they scatter more.

\subsubsection{Replacement of STeEl IN PFZ DEVICE BY ANOTHER METAL}

As can be seen in Fig. 6, putting steel material away from our experimental setup would have a big influence on reducing the amount of scattered neutrons.

We performed simulations where steel was replaced by another considerable metal. Firstly, we tried to replace steel by aluminum.

With respect to relative error of our simulation which was $2 \%$, we may not say that replacement of steel by aluminum would have any remarkable effect on reducing of amount of scattered neutrons. We made the same conclusion for case when steel was replaced by copper. 
Neutron energy spectrum in place of probe

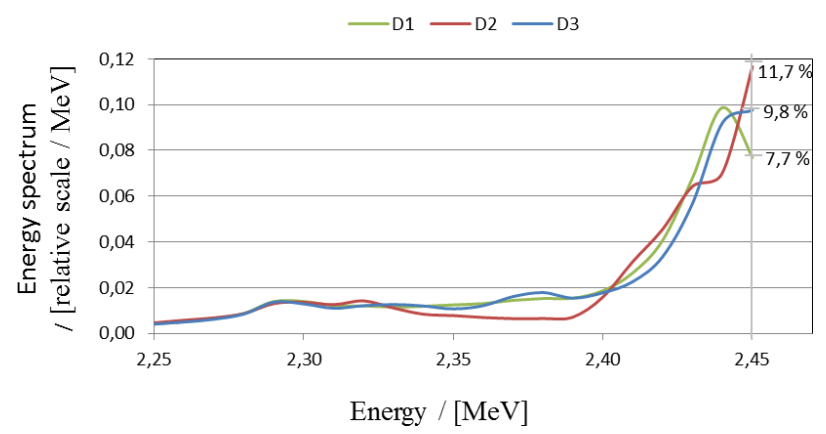

FigURE 5. We can see what percentage of neutrons remained unscattered during flight to probes D1, D2 and D3.

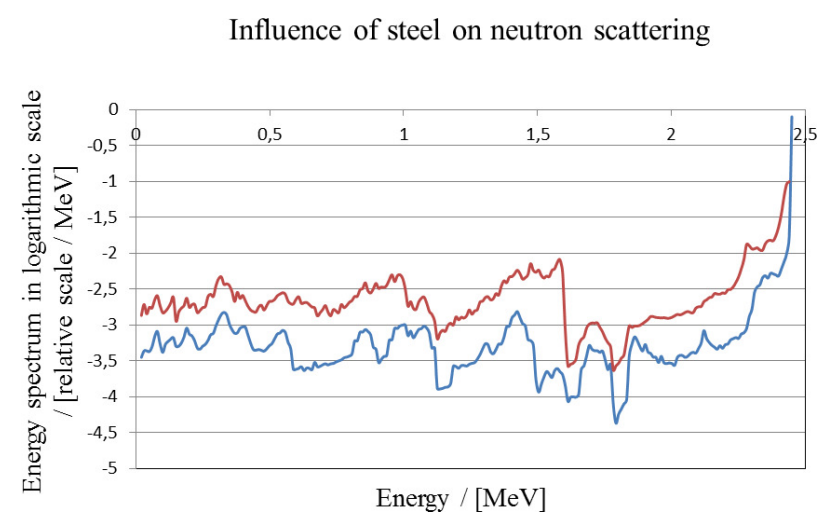

Figure 6. Red line - complete experimental setup, blue line - experimental setup without steel.

Replacement of steel by aluminum

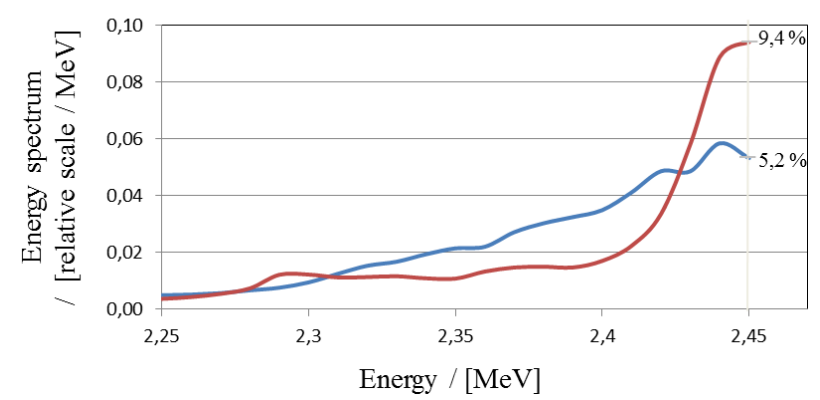

Figure 7. Red line - experimental setup with steel, blue line - steel replaced by aluminum.

\subsubsection{Correspondation BETWEen Simulation AND EXPERIMENT}

Naturally, there is a question whether our computer simulations correspond with reality of the experimental setup. Neutron energy spectrum in results of our simulations may not correspond with measured neutron energy spectrum because the physics of generating of neutrons may be much more complicated in reality than we supposed in our simulation. Specifically, it is especially choice of mono-energetic point

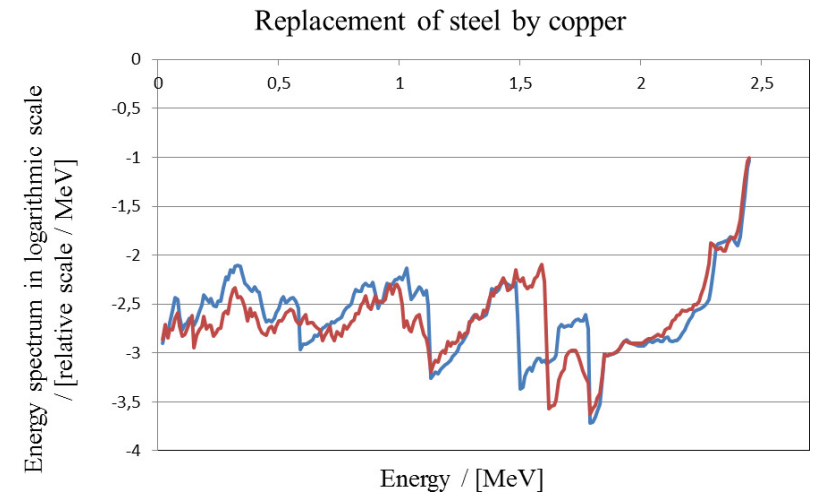

FiguRE 8. Red line - experimental setup with steel, blue line - steel replaced by copper.

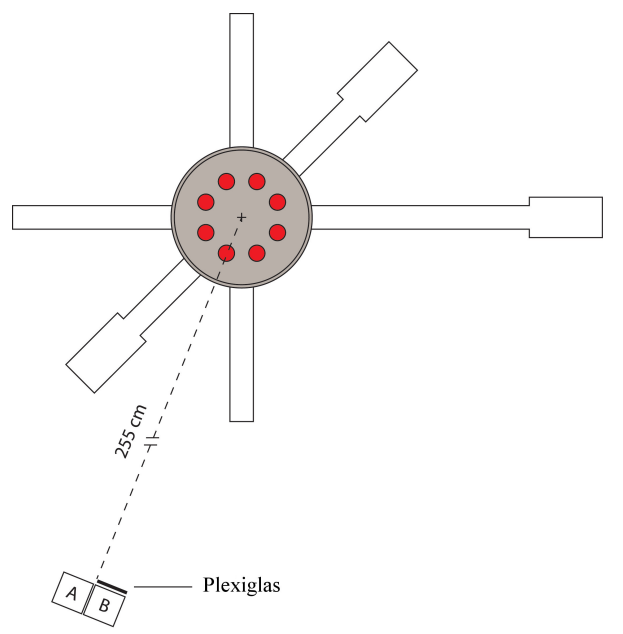

Figure 9. Experimental setup with shielded and unshielded probe.

source in our input fil€ However, we can make experiment where we change experimental setup in a way that we observe change in measured neutron spectrum, and also make computer simulation which has implemented the same change in parameters of the input file. We realized this idea so that we put two probes next each other in experiment, and in front of one of them we placed thin plexiglas desk.

In shielded probe A we measured smaller area $X$ under neutron signal than in unshielded probe B.

In computer simulation, we can find equivalents $Y_{\mathrm{A}}$ and $Y_{\mathrm{B}}$ to areas $X_{\mathrm{A}}$ and $X_{\mathrm{B}}$. We estimated value of $X_{\mathrm{A}} / X_{\mathrm{B}}$ and $Y_{\mathrm{A}} / Y_{\mathrm{B}}$ as

$$
\frac{X_{\mathrm{A}}}{X_{\mathrm{B}}}=1.7 \pm 0.3 \quad \text { and } \quad \frac{Y_{\mathrm{A}}}{Y_{\mathrm{B}}}=1.3 \pm 0.1 .
$$

This result is in favor of the hypothesis that our simulations match quite reality.

\footnotetext{
${ }^{1}$ Nevertheless, for our purposes when we compare scattering of neutrons flying in specific direction, it is useless to consider non-isotropic distribution of neutrons that is surely present in our experiment.
} 


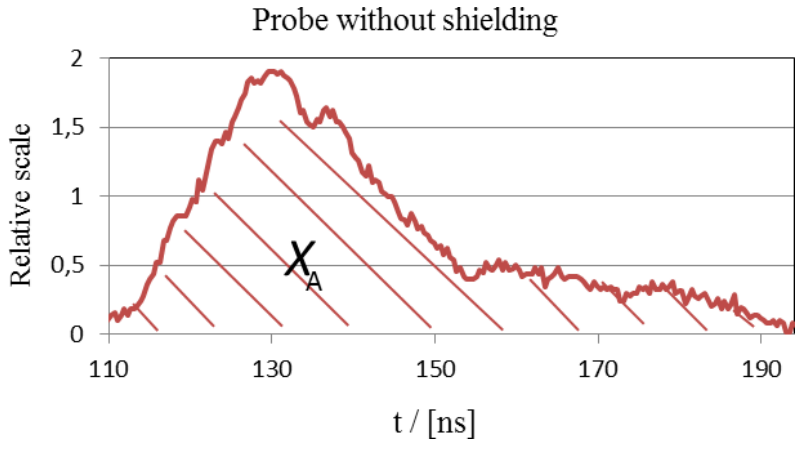

Figure 10. Area under neutron signal of unshielded probe.

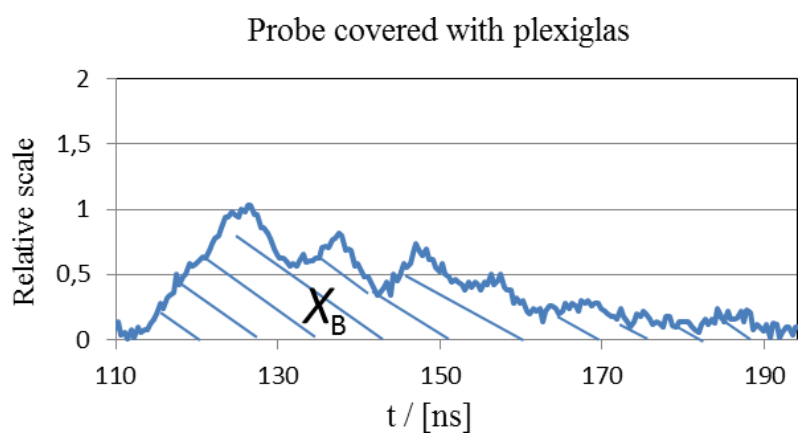

Figure 11. Area under neutron signal of shielded probe.

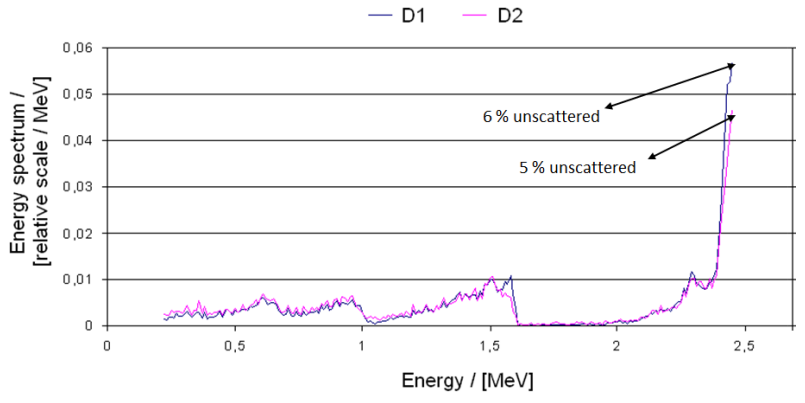

Figure 12. Neutron energy spectrum in place of two scintillation probes.

\subsection{MCNP RESUlTS FOR GIT-12 DEVICE}

We simulated neutron energy spectrum in place of two scintillation probes, one placed axially $10.12 \mathrm{~m}$ above neutron source (D1), and one radially in the same distance (D2). Relative inaccuracy of our results was $0.2 \%$.

Probe D2 is placed near great boxes with Marx generators which are filled with oil. Oil may have greater influence on scattering of neutrons entering probe D2, so we removed it from our simulation and observed how neutron energy spectra changed.

If we remove oil in boxes, we get approximately $1 \%$ greater amount of unscattered neutrons in D2. This leads us to conclude that there is approximately the same amount of scattered neutrons flying in axial and radial direction if we remove oil from our experimental setup.

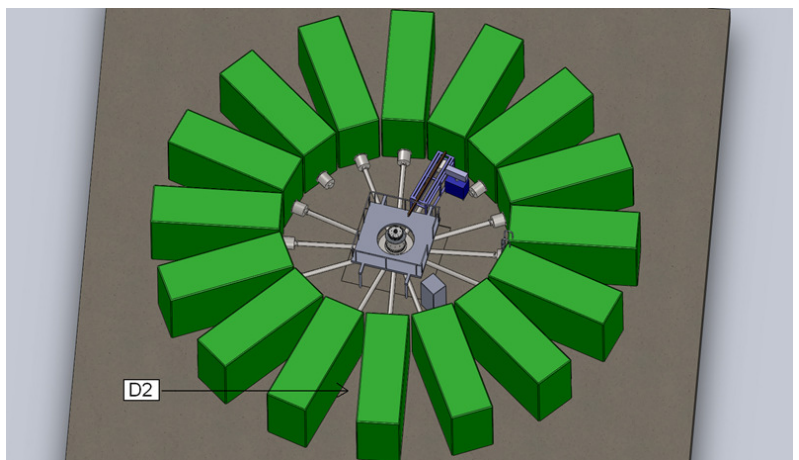

Figure 13. Position of probe D2 which is situated between boxes with Marx generators (green blocks).

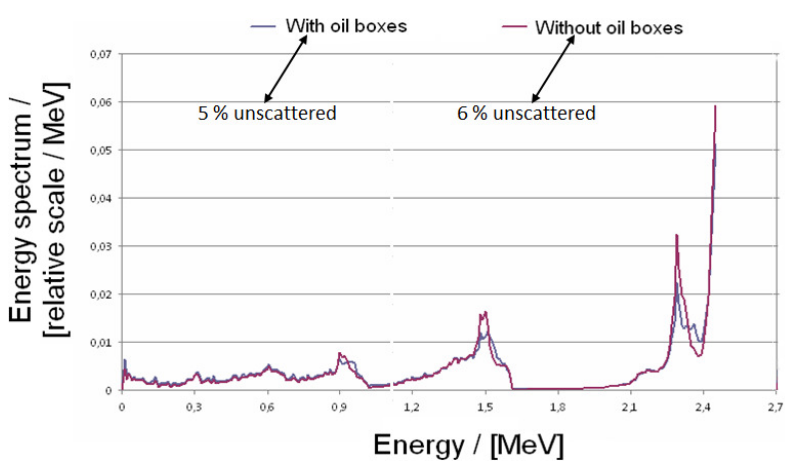

FiguRE 14. Neutron energy spectrum in place of probe D2; we consider the case of removing scattering medium from our experimental setup.

\section{Conclusion}

\subsection{Summary of the Results at PFZ DEVICE}

The greatest amount of scattered neutrons fly in axial direction. We see the reason of it in fact that they have to overcome substantially thicker layer of metal.

Steel used in our device seems to be convenient material for our purposes - in our simulations, other metals would have the same or greater influence on scattering of neutrons.

Comparing simulation with experiment gave us feedback which confirmed our hypothesis that our simulations correspond with reality.

\subsection{Conclusions for GIT-12 Device}

There is $1 \%$ less scattered neutrons flying to axial probe than to radial probe.

Our results showed that this difference may be caused by boxes with oil which are placed near radial probe.

\section{ACKNOWLEDGEMENTS}

This work was supported by the Research Program MSMT No. LA08024, ME09087 and GACR P205/12/0454, IAEA RC 14817 and CTU SGS 10/266/OHK3/3T/13.

\section{REFERENCES}

[1] D. Klir. The Study of a Fibre Z-pinch. CTU FEE, Prague, 2005. Doctoral thesis. 
[2] D. Klir, P. Kubes. Neutron emission generated during wire array Z-pinch implosion onto deuterated fiber. Physics of Plasmas 15(1-13), 2008.

[3] M. Kralik, J. Krasa. Application of a Bonner sphere spectrometer for determination of the energy spectra of neutrons generated by $\approx 1 \mathrm{MJ}$ plasma focus. Review of Scientific Instruments 81(1-5), 2010.
[4] J. Krasa, M. Kralik. Anisotropy of the emission of D-D fusion neutrons caused by the plasma-focus vessel. Plasma Physics and Controlled Fusion 50(1-10), 2008.

[5] O. Sila. Energy spectrum of neutrons from $D(d, n) 3 H e$ reactions in plasma focus discharge. CTU FEE, Prague, 2011. 